\title{
Statistical and Stochastic Modeling of Gang Rivalries in Los Angeles
}

\author{
Mike Egesdal ${ }^{* \dagger} \quad$ Chris Fathauer ${ }^{\ddagger} \quad$ Kym Louie $^{* \ddagger}$ \\ Jeremy Neuman ${ }^{\dagger}$ \\ Advisors: \\ George Mohler ${ }^{\S}$ and Erik Lewis ${ }^{\dagger}$
}

\begin{abstract}
Gang violence has plagued the Los Angeles policing district of Hollenbeck for over half a century. With sophisticated models, police may better understand and predict the region's frequent gang crimes. The purpose of this paper is to model Hollenbeck's gang rivalries. A self-exciting point process called a Hawkes process is used to model rivalries over time. While this is shown to fit the data well, an agent based model is presented which is able to accurately simulate gang rivalry crimes not only temporally but also spatially. Finally, we compare random graphs generated by the agent model to existing models developed to incorporate geography into random graphs.
\end{abstract}

\section{Introduction}

Gang violence is a prevalent problem in the Los Angeles policing district Hollenbeck [13]. Just east of downtown, it is among the top three most violent Los Angeles policing regions, despite having a total area of just 15.2 square miles [13]. Gang related crimes are not a new problem in Hollenbeck; some of the gangs have existed since before WWII [13]. Thus heated battles over territory are a deeply rooted phenomenon and central to the gang related violence that plagues Hollenbeck every day.

*Corresponding author: kym.louie@gmail.com, egesdal@gmail.com

$\dagger$ University of California, Los Angeles

¥Harvey Mudd College

§Sponsor: gmohler@math.ucla.edu 


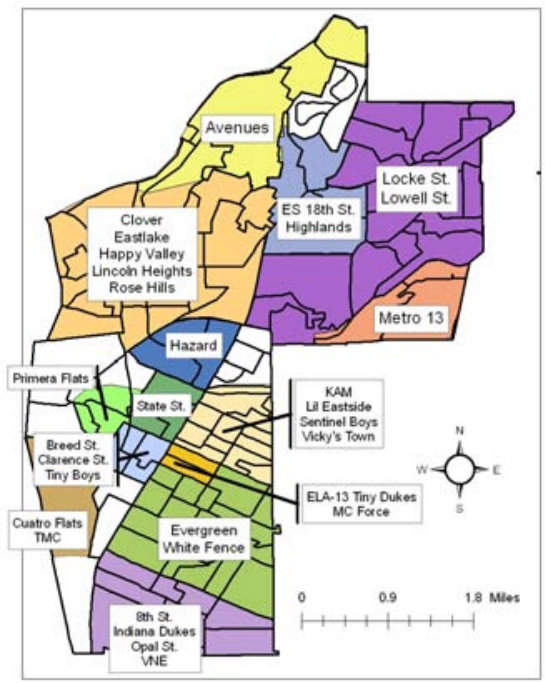

Figure 1: Map of Hollenbeck's census block groups and gang territories.

Police data on gang crimes from 1999 to 2002 reveal a plethora of gangs in Hollenbeck. This work only considers the 33 gangs that have committed over four crimes against other gangs in this time period.

Gang rivalries are analyzed by modeling their behavior through time and space. In Section 1, a self-exciting point process called a Hawkes process is presented to model rivalry behavior over time. This point process has been used in seismology to model the rate of earthquakes occuring; the rate jumps up following an event as one expects aftershocks. In our case, the expected aftershocks are the retaliations following gang crimes.

While the Hawkes process is shown to fit the data better than the memoryless Poisson process, an ideal model can mimic both the temporal and spatial distributions of a rivalry's crimes. To accomplish this, in Section 2 an agent based model is used to simulate gang activity. Agent based models have been used in a vast number of subjects, including biology and epidemiology [5][12][15]. Such a model is applicable to our problem because it allows for gangs to have individual gang members moving with bias toward other gangs, fighting and building rivalries. This model is shown to closely replicate actual gang behavior. Finally, graphs from the agent based model are compared to a random method of connecting nodes called Geographical Thresholding. The method incorporates the distance between nodes and randomly assigned weights to decide whether or not to make a connection. 


\section{Data}

The data we use in producing our models were provided by the Los Angeles Police Department: 1200 gang related violent crimes that occurred between 1999 and 2002. Each entry lists the latitudinal and longitudinal coordinates, the victim gang, the suspected gang, the type of crime, and the date and time of the incident. As the focus of this study is gang rivalries, events were removed from the data set when only one gang was involved or when incomplete information was available.

\section{Hawkes Process}

\subsection{Motivation}

The Hawkes process is a self-exciting point process commonly used in seismology to model earthquakes. There is a background rate at which events can occur; once an event does occur, the rate jumps up as aftershocks are expected to follow the initial event. In the case of gangs, the expected aftershocks are the retaliations that follow gang crimes. The Hawkes process is compared to the Poisson process and shown to be, in general, a better fit to the data. Attention is restricted to crimes between specific pairs of gangs, as the activities of these pairs drive the dynamics of the system. Only rivalries with more than ten crimes over the three year period from 1999 to 2002 are considered, since parameter estimation based on too few crimes may be inaccurate.

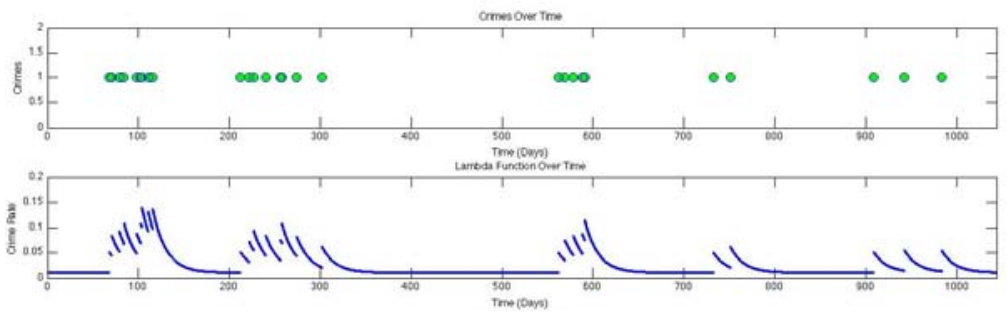

Figure 2: A plot of the Locke-Lowell rivalry's crimes over time with the respective Hawkes process rate function, $\lambda(t)$.

\subsection{Equations}

The rate function for the Hawkes process is:

$$
\begin{array}{cl}
\lambda(t)=\mu+k_{0} \sum_{t>t_{i}} g\left(t-t_{i} ; w\right) \\
\begin{array}{|ll}
\mu & \text { Background Rate } \\
k_{0} & \text { Scaling Factor } \\
w & \text { Rate of Decay } \\
\hline
\end{array}
\end{array}
$$


The rate function $\lambda(t)$ is the expected rate at which events occur. $\mu$ is the background rate at which events can occur, $k_{0}$ is a scaling factor for the effect of a crime, and $w$ is the rate of decay for a crime's effect [9]. For the function $g\left(t-t_{i} ; w\right)$ we used an exponential distribution, $w e^{-w\left(t-t_{i}\right)}$. A higher $\mu$ increases the overall rate of crimes. A higher $k_{0}$ increases the jump in the rate function following a crime. A smaller $w$, and thus a slower decay, means that the rate stays elevated longer following a crime. When the rate is elevated, more crimes are likely to happen, which in turn increases the rate further. Hence, the process is self-exciting.

The parameters for the Hawkes process are obtained using maximum likelihood estimation. The log likelihood function is:

$$
\log \mathrm{L}=\sum_{i=1}^{N} \log \lambda\left(t_{i}\right)-\int_{0}^{T} \lambda(t) d t
$$

\begin{tabular}{|ll|}
\hline $\mathrm{L}$ & Likelihood Function \\
$N$ & Number of Events \\
$T$ & Total Time (Days) \\
\hline
\end{tabular}

The $\log$ likelihood function is maximized with respect to $k_{0}, w$, and $\mu$ [9]. In the case of our police data, it is fit between a pair of gangs. Each $t_{i}$ is the time of a crime, measured to the hundredth of a day, between the two gangs. Built into the log likelihood function is the constraint that $\lambda(t)$ integrates to $N$. The total time window of our crime data, $T$, is 1043.6 days. Due to the nature of nonlinear maximization, different sets of parameters can be obtained when maximizing the function, based on the starting point given. One way to check the accuracy of the parameters is to see that $\lambda(t)$ does indeed integrate to $N$. Many different starting points are experimented with to find the parameters that both produce the highest log likelihood value and let $\lambda(t)$ integrate exactly to $N$.

\subsection{Model Comparison}

In this section, we first point out the predictive nature of the Hawkes process, as one can run simulations with a rivalry's parameters. We then compare the Hawkes process to the Poisson process using both a histogram analysis [10] and the Akaike Information Criterion (AIC) [1]. To illustrate our methods, we present figures for the rivalry between the Locke Street and Lowell Street gangs. These two gangs committed 27 violent crimes against each other in a period of 1043.6 days from 1999 to 2002. MLE parameters are obtained as described in Section 1.2. We summarize our findings with a table for the nine rivalries with at least ten crimes. 


\subsubsection{Simulations}

Once parameters are obtained for a rivalry, one can run simulations to obtain a rough prediction of the rivalry's behavior in the future.
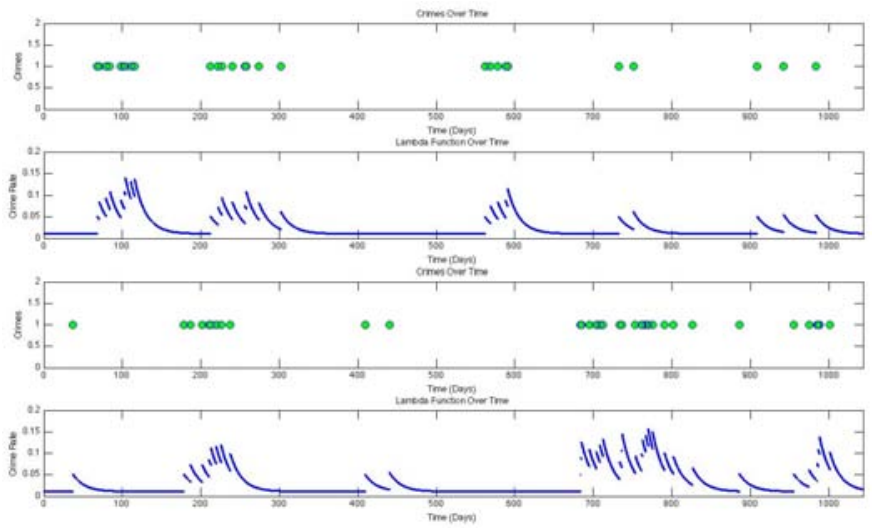

Figure 3: On top, a plot of the Locke-Lowell rivalry's crimes over time with the respective rate function, $\lambda(t)$. On bottom, simulated crimes from a Hawkes process with the Locke-Lowell rivalry's parameters and the corresponding rate function, $\lambda(t)$.

\subsubsection{Histogram Analysis}

In this section we examine all inter-attack times $t_{i}-t_{j}$ such that $i>j$. We again use the Locke-Lowell rivalry to illustrate our methods. If the data come from a Poisson process, then a histogram of inter-attack times will be roughly uniform when looking at a short inter-attack time window (e.g. crimes less than 90 days apart) due to minimal finite window effects [10]. This is because, with an infinite window, inter-event times from a Poisson process are uniformly distributed.

If the data come from a Hawkes process, there will be high frequencies for short inter-attack times (e.g. crimes less than 30 days apart) due to selfexcitation [10]. The reason that self-excitation is expected is that gang crimes are followed by retaliations. These high frequencies should decay quickly and then become somewhat uniform or start to decay linearly due to finite window effects [10]. 

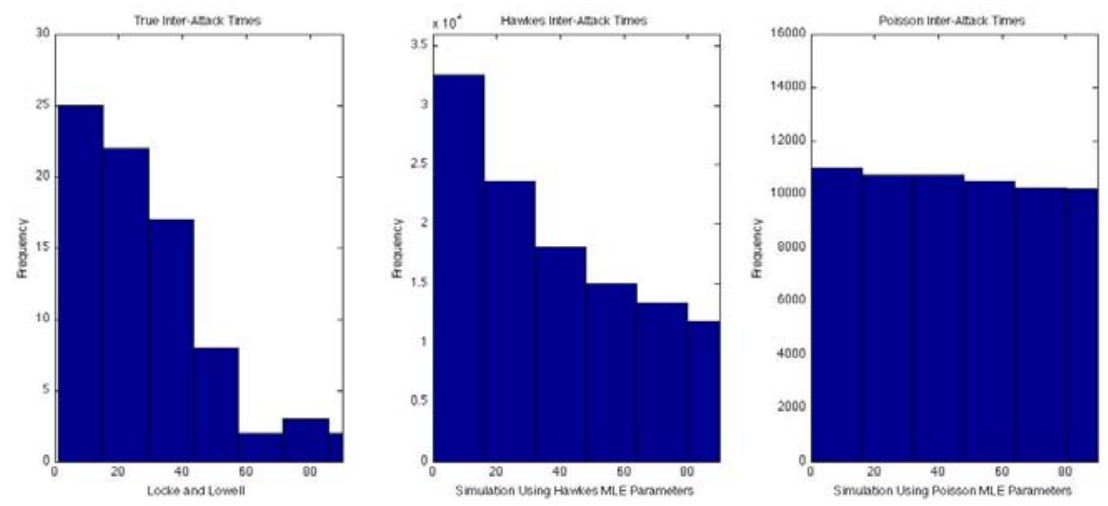

Figure 4: A histogram of Locke-Lowell inter-attacks times for the true data and model simulations.

Above are the true inter-attacks times for the Locke-Lowell rivalry (left), inter-attack times for 1000 Hawkes simulations with the rivalry's MLE parameters (middle), and inter-attack times for 1000 Poisson simulations with its MLE parameter for the rivalry (right). The purpose of running 1000 simulations is to capture the true distribution shape of each model with its respective MLE parameters for the rivalry. It is the distribution shape over a fixed time window that matters here, not the vertical axis scale. The time window is 90 days, hence there should be minimal finite window effects. The histogram distribution matches that of the Hawkes process much more closely than that of the Poisson process. Due to the small number of data for each rivalry, the simulation parameters are fit to the data used for comparison as is done in Short et al [10]. If more data were available, a better comparison would be to fit the parameters to half of the data set and then compare it with the other half.

\subsubsection{Akaike Information Criterion}

We also compare the Hawkes process to the Poisson process using the Akaike Information Criterion. It is defined as

$$
\mathrm{AIC}=2 k-2 \log \mathrm{L}
$$

where $\mathrm{k}$ is the number of parameters in the model and $\log \mathrm{L}$ is the value of the maximized log likelihood function [1]. The Akaike Information Criterion gives values to rank models such that the model with a lower AIC value is the better fit [1]. This criterion penalizes models for having more parameters; however, for the rivalries we examine the AIC generally supports the Hawkes process with three parameters over the Poisson process with only one parameter.

This simple formula is derived by $\mathrm{H}$. Akaike as follows: consider a maximum likelihood problem over a family of models $f(x \mid \theta)$ with a vector parameter $\theta$, true parameter $\theta_{0}$ and $n$ observations. Let $\mathrm{J}$ be the (positive definite) Fisher 
information matrix, which gives a norm \|\|$_{J^{2}}$. When $\theta$ is restricted to vary within a smaller $k$-dimensional subspace $\Theta$, the distribution of $n *\left\|\theta-\theta_{0}\right\|_{J^{2}}$ is, under certain conditions, a chi-square distribution with $k$ degrees of freedom [1]. It can then be shown that $n *\left\|\hat{\theta}-\theta_{0}\right\|_{J^{2}}+k=E\left[2 n * I\left(\theta_{0} ; \hat{\theta}\right)\right]$, where $I\left(\theta_{0} ; \hat{\theta}\right)$ is the Kullback-Leibler mean information. Akaike claims that one should choose the model that minimizes $E\left[I\left(\theta_{0} ; \hat{\theta}\right)\right][1]$. Noting this, one would then want an approximation of $n *\left\|\hat{\theta}-\theta_{0}\right\|_{J^{2}}$. It can be shown that $2\left(\sum_{i=1}^{n} \log f\left(x_{i} \mid \theta_{0}\right)-\right.$ $\left.\sum_{i=1}^{n} \log f\left(x_{i} \mid \hat{\theta}\right)\right)$ is such an approximation of $n *\left\|\hat{\theta}-\theta_{0}\right\|_{J^{2}}$, but has a downward bias which may be corrected by adding $k$ [1]. The first sum in this approximation may be ignored for comparison between two models, since it is the same for both, and the second term is precisely the log likelihood evaluated at $\hat{\theta}$. This leads to the simple formula for the Akaike Information Criterion, $2 k-2 \log \mathrm{L}$.

\subsubsection{Hawkes Summary Table}

\begin{tabular}{|c|c|c|c|c|c|c|c|}
\hline \multicolumn{7}{|c|}{ Hawkes Summary Table } \\
\hline Rivalry & Poisson $\mu$ & $k_{0}$ & $w$ & $\mu$ & Poisson AIC & Hawkes AIC & Best Fit \\
\hline Loc-Low & 0.026 & 0.584 & 0.068 & 0.011 & 253.3 & 242.2 & Hawkes \\
Clo-Eas & 0.032 & 0.209 & 0.921 & 0.025 & 296.0 & 288.8 & Hawkes \\
Lin-Eas & 0.014 & 0.121 & 0.096 & 0.013 & 159.3 & 162.7 & Poisson \\
KAM-Sta & 0.018 & 0.259 & 0.101 & 0.014 & 192.2 & 193.1 & Poisson \\
Tin-Sta & 0.015 & 0.124 & 28.6 & 0.013 & 167.7 & 157.6 & Hawkes \\
MCF-ELA & 0.017 & 0.513 & 0.080 & 0.008 & 184.2 & 173.6 & Hawkes \\
VNE-Opa & 0.019 & 0.390 & 0.134 & 0.012 & 200.2 & 192.8 & Hawkes \\
VNE-8th & 0.015 & 0.488 & 0.033 & 0.008 & 167.7 & 167.0 & Hawkes \\
TMC-Cua & 0.025 & 0.074 & 0.021 & 0.023 & 246.0 & 247.6 & Poisson \\
\hline
\end{tabular}

Figure 5: Hawkes Summary Table For Nine Largest Rivalries

In the table above, Poisson $\mu$ is the maximum likelihood estimate of fitting a Poisson process to the data; for a Poisson process this estimate is the average number of events per day over the time period. In general, the parameters found for the rivalries were of a similar magnitude. However, despite many starting seeds for the nonlinear maximization, the best set of parameters for the Tiny Boys - State Street rivalry gives a $w$ of 28.6. These parameters do allow the rate function to integrate exactly to $N$.

\subsection{Summary}

The preceding analysis shows that the Hawkes process generally fits the temporal distribution of gang rivalry crimes better than the Poisson process. While there are a couple of rivalries that have crimes spread more or less independently through time, most rivalries have significant clustering of attacks. 


\section{Agent Based Model}

The problem of simulating actions of many gang members is approached using an agent based model, referred to as the random walker program. In agent based models, each agent acts individually following a defined set of rules. The agents move randomly with a bias such that the group exhibits the behavior of interest. Agent based models have been used to gain insight into behavior and population dynamics of humans and animals [5][11][12][15].

\subsection{The Program}

The random walker program simulates gang members moving in a semirandom manner on a map. In this program, each "agent" represents a group of gang members, as stereotypically gang members operate in groups. The map with anchor points, blocked locations, and any other static features is referred to as the environment. In order to determine what occurs each time step, the program calls four functions.

\subsubsection{Moving Gang Members}

The first function moves all the gang members to their next location. Each member looks at the spaces above, to the left, to the right, and below them, as well as the space the are occupying and makes a weighted random choice for which direction to move.

The probability that a gang member $g$ moves to space $s$ from space $s_{0}$ is

$$
P(g \rightarrow s)=\frac{W(s, g, t)}{\sum_{s_{0}^{\prime} \sim s_{0}} W\left(s_{0}^{\prime}, g, t\right)},
$$

where $s_{0}^{\prime} \sim s_{0}$ indicates all cells neighboring $s_{0}$ and $W(s, g, t)$ is the weight of space $s$ for gang member $g$ at time step $t$. This is found by

$$
W(s, g, t)=W^{0}+C \sum_{i} e^{\frac{-\left(s_{x}-x_{i}\right)^{2}-\left(s_{y}-y_{i}\right)^{2}}{2 \sigma^{2}}}+D e^{\frac{-\left(s_{x}-\bar{x}\right)^{2}-\left(s_{y}-\bar{y}\right)^{2}}{2 \hat{\sigma}^{2}}},
$$

where $\left\{\left(x_{i}, y_{i}\right)\right\}$ is the set of all locations of gang members of the target gang and $(\bar{x}, \bar{y})$ is the target gang's center. $W^{0}$ is the base weight for all cells. D was usually taken to be 0 or nearly 0 such that movement depended entirely or almost entirely on the locations of other agents.

\subsubsection{Adding New Gang Members}

The next function called adds new gang members. Gang members are added at anchor points and then move normally at the next time step. An anchor 
point is a centralized location from which criminals tend to stage their crimes. These were taken to be the average location of all crimes identified with the gang. Gang members are added to the map at a rate $\Gamma$, such that

$$
P(\text { new gang member appears for gang } \mathrm{G})=\Gamma \text {. }
$$

When a gang member is added, he randomly selects another gang to target. This is biased such that he is more likely to target a gang with which his gang has a higher rivalry strength. Once a target gang is selected, the gang member never changes his target.

The target gang for gang $H$ is selected as

$$
P\left(\text { target }=G_{i}\right)=\frac{\mathrm{R}_{H G_{i}}(t)}{\sum_{j} \mathrm{R}_{H G_{j}}(t)},
$$

summing over all gangs.

\subsubsection{Fights Between Gang Members}

Once new gang members have been added, the program checks to see if any gang members are occupying the same cell. If so, each cell that has multiple gang members in it can currently support up to one fight. For a cell with multiple gang members in it, the function looks at random pairs of gang members occupying the cell until either a pair fights or until all pairs have been examined. Each cell only supports one fight because it is assumed that after a crime has occurred, police appear to prevent any further crimes from occurring that time step. However, depending on the length of the time step used, it is sometimes more reasonable to let cells support multiple fights.

The probability that two gang members fight is dependent on the rivalry strength between the two gangs that they belong to $\left(G_{1}\right.$ and $\left.G_{2}\right)$ :

$\rho_{g_{1} g_{2}}(t)= \begin{cases}1-e^{-\mathrm{R}_{G_{1} G_{2}}(t) \delta t} & \text { no fights have yet occurred here in time step } t \\ 0 & \text { otherwise }\end{cases}$

This is very similar to the function used by Short et al. in a study of burglaries to determine whether their agents burgled houses [11]. Gang members never attack members from their own gang.

\subsubsection{Updating Rivalry Strengths}

The final function called each iteration updates the rivalry strengths. The rivalry strengths decay over time and are increased by any fights that have occurred in the current time step. This is very similar to the function used by Short et al. to determine attractiveness of houses to burglars [11]. Rivalry 
strengths are determined as a base rivalry strength plus a function that varies as stated above.

$$
\mathrm{R}_{i j}(t)=\mathrm{R}^{0}+\mathrm{B}_{i j}(t),
$$

where $\mathrm{B}_{i j}(t)$ is defined

$$
\mathrm{B}_{i j}(t+\delta t)=\mathrm{B}_{i j}(t)(1-\omega \delta t)+\theta \mathrm{E}_{i j}(t) .
$$

$\omega$ determines how fast the rivalry strength decays, $\theta$ sets how much a fight between two gangs increases the rivalry strength, and $E_{i j}(t)$ is the amount of fights that have occurred between gang members of gangs $i$ and $j$.

These are the variables and main functions that appear:

\begin{tabular}{|c|c|}
\hline \multicolumn{2}{|c|}{ Variables and Functions of Agent Based Model } \\
\hline$W(s, g, t)$ & Weight of space s for gang member $\mathrm{g}$ \\
$\sigma$ & Determines how distance between spaces affects weights \\
$\Gamma$ & Rate of gang member generation per gang per time step \\
$\rho_{i j}(t)$ & Probability that gang member i and gang member $\mathrm{j}$ fight \\
$\mathrm{R}_{i j}(t)$ & Rivalry strength between gangs i and $\mathrm{j}$ \\
$\delta t$ & The length of time per timestep \\
$\omega$ & Determines the rate of decay of rivalries \\
$\theta$ & Increase in rivalry strength per attack \\
$\mathrm{E}_{i j}(t)$ & Attacks between gangs i and $\mathrm{j}$ in timestep t \\
\hline
\end{tabular}

Figure 6: Variables and Functions of Agent Based Model

\subsection{Parameters}

The parameters that can be changed in the simulation to affect its behavior are:

- Parameters affecting rivalry strength $\mathrm{R}$

- A high $\mathrm{R}$ (such that $\mathrm{R} \delta t>3$ ) corresponds to a $\rho_{i j}=0.95$, so any strengths higher than that are approximately equivalent in that they both will cause fights to occur with probability nearly 1 .

- Base rivalry strength $\mathrm{R}^{0}$

* Increasing/decreasing $\mathrm{R}^{0}$ increases/decreases the likelihood that two gangs will fight upon encountering each other, independent of the amount of fights that have previously occurred between them.

- Amount rivalry strength is increased per fight $\theta$

* Increasing $\theta$ increases the amount of time and extent to which a fight significantly affects rivalry strength. If $\theta$ is large and $\mathrm{R}$ is small, a fight greatly increases the probability of future fights 
occurring. However, if $\mathrm{R}$ is large, it does not significantly increase the probability of a fight, but affects how long $\rho_{i j} \approx 1$ due to this fight.

- Decay of rivalry strength $\omega$

$\star$ Increasing/decreasing $\omega$ decreases/increases the time required for $\mathrm{R}$ to decay to approximately $\mathrm{R}^{0}$. $\omega$ should always be set between 0 and 1.

- Parameters affecting space weighting $W$

- Base weight $W^{0}$

$\star W^{0}$ must always be nonzero in order to allow gang members to choose spaces when they cannot "see" any of their targets, but it is usually made to be very small $\left(10^{-6}\right)$. Increasing $W^{0}$ sends gang members in more random directions with less tendency towards their targets.

- Coefficients $C$ and $D$

* The actual values of $C$ and $D$ do not matter. The ratio $C / D$ is similar to setting how important the locations of gang members are relative to the importance of the anchor points as targets. Setting both to 0 will cause unbiased random motion.

- "Sight" range $\sigma$

* Increasing/decreasing $\sigma$ increases/decreases how far a gang member can "see", or how fast a weight due to a given target decays over distance. A greater $\sigma$ enables a gang member to target more distant locations.

- Rate at which gangs members are added $\Gamma$

- Increasing/decreasing $\Gamma$ increases/decreases the rate at which gang members are added to the map. Because over time this program reaches an equilibrium for number of active gang members for nearly all parameter values, increasing/decreasing $\Gamma$ increases/decreases the number of fights that occur each turn.

- Length of time step $\delta t$

- Sets how much time a time step represents. $\delta t$ must be set such that $\Gamma$ makes sense for one time step. Changing $\delta t$ allows the simulation to run for more time steps per unit time or cover a greater period in fewer steps. 


\subsection{Model Comparison}

\begin{tabular}{|c|c|c|}
\hline \multicolumn{3}{|c|}{ Agent Based vs. Hawkes Process Table } \\
\hline Agent Based & Hawkes Process & Role In Model \\
\hline$\omega$ & $w$ & Rate of Decay for Rivalry Intensity \\
$\mathrm{R}_{0}$ & $\mu$ & Background Intensity of Rivalry \\
$\theta$ & $k_{0}$ & Jump In Intensity Following Crimes \\
$\mathrm{R}_{i j}(t)$ & $\lambda(t)$ & Rivalry Intensity Function \\
\hline
\end{tabular}

Figure 7: Agent Based vs. Hawkes Process Table

As seen in the table above, there are many qualitative similarities between the Hawkes process and the agent based model. For example, both $\omega$ and $w$ determine the rate of the rivalry intensity's exponential decay.

\subsection{Temporal Analysis}

In this section, we just consider a pair of gangs to simplify the analysis of rivalry attacks over time. All parameters are obtained from experimentation. Further research may consider the temporal distribution of crimes with many gangs, and may explore more sophisticated methods of obtaining parameters.

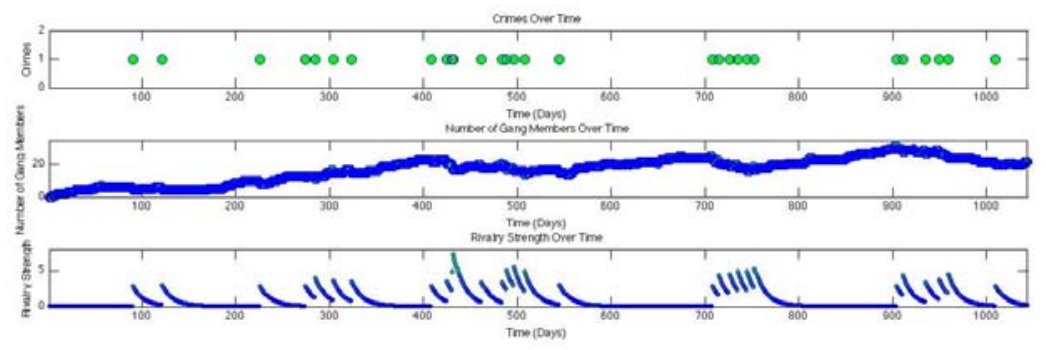

Figure 8: Temporal Analysis for Agent Based Model

Above is a temporal graph of a rivalry's crimes generated from the agent based model (top), a graph of the number of active gang members through time (middle), and a graph of the rivalry strength function (bottom). As mentioned earlier, the number of gang members reaches a stable equilibrium as gang members are taken off the map through fights and enter the map with a fixed probability each time step. 


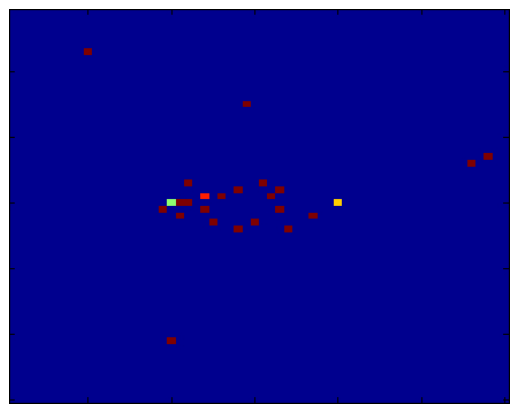

Figure 9: Two gangs in a rivalry.

The rivalry strength function is built to act like the Hawkes process rate function, even though they are implemented differently:

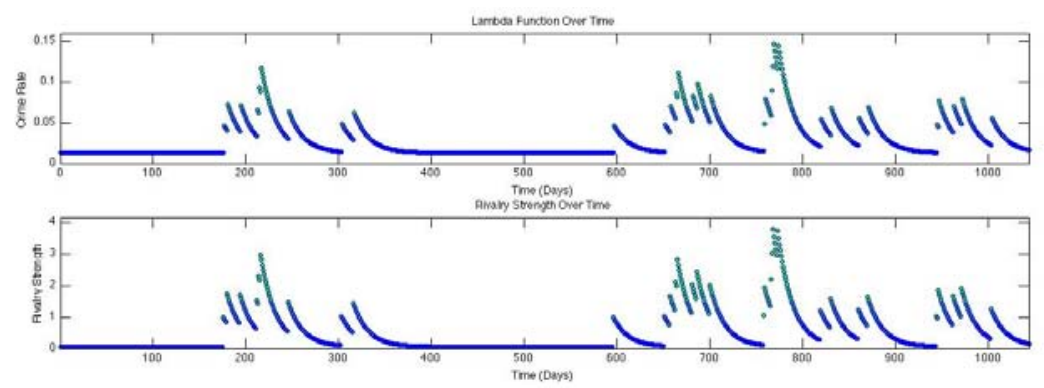

Figure 10: Hawkes Rate Function $\lambda(t)$ vs. Rivalry Strength function $\mathrm{R}(t)$

The above graph is provided to help visualize the qualitative similarities between the Hawkes rate function and the rivalry strength function, not to suggest a more significant relationship between the two. We used maximum liklihood estimation for the data produced by the agent model to estimate parameters for the Hawkes process. The rate function $\lambda(t)$ is plotted for the generated crimes below. The graph of $\lambda(t)$ is similar to the graph of the rivalry strength function $\mathrm{R}(t)$. Despite the different implementations of the two functions, this suggests that the way in which the agent based model generates crimes resembles a Hawkes process. 

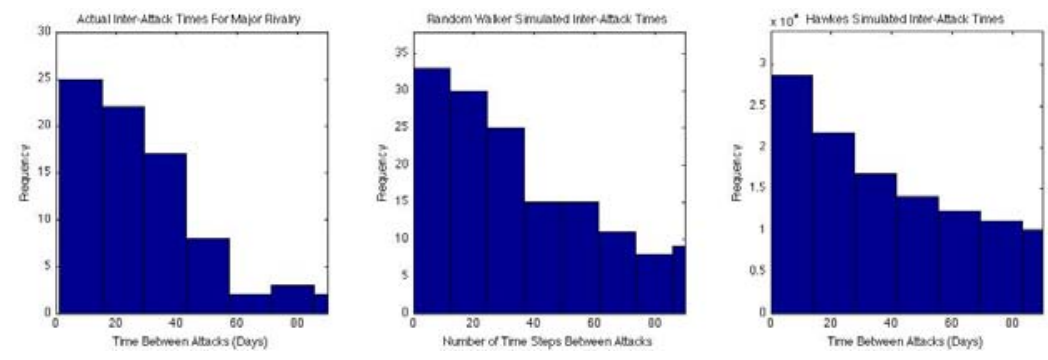

Figure 11: Histograms of the Locke-Lowell rivalry's inter-attack times (left), agent model simulated crimes with experimental parameters (middle), and Hawkes simulated crimes using the rivalry's MLE parameters (right)

As seen above, the inter-attack times from the agent based model are qualitatively similar to both our actual data and that of a Hawkes process. The inter-attack times from the agent based model are hardly uniform over this short time window of 90 days, as would be expected if the events were from a Poisson process [10]; with a total time window of 1044 time steps, there should be minimal finite window effects. In summary, the agent based model's generation of rivalry attacks is similar to the Hawkes process.

\subsection{Spatial Analysis}

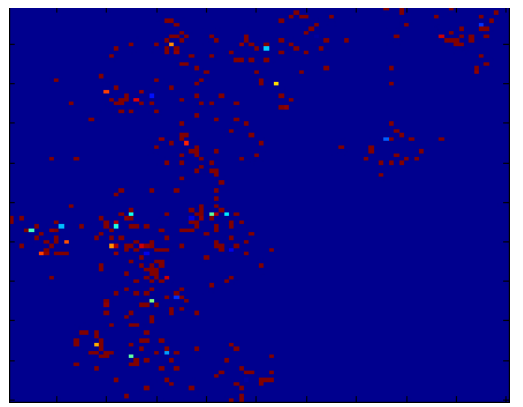

Figure 12: All Hollenbeck gangs and agents scaled to 100x100 grid.

A vital part of studying our agent based model centers around the spatial distribution of crimes. Specifically, spatial plots of the total crimes, crimes specific to a single gang, and inter-rivalry gang crimes are analyzed. In general, this model reflects where gang crimes are likely to occur in Hollenbeck.

\subsubsection{Gang Anchor Points}

The method we use to construct an anchor point for a specific gang in this model is averaging all of the coordinates of all of the reported crimes identified 


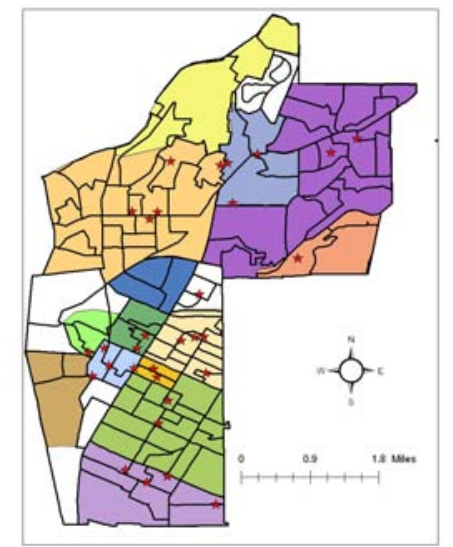

Figure 13: Plot of Average Coordinates for Each Gang

with this gang. Some of the averages are skewed a little due to outlier crimes, and some of the averages may be pulled slightly towards the center of Hollenbeck (Figure 13). This method of creating anchor points yields results similar agreeable with previously established anchor point estimates from the LAPD.

\subsubsection{Spatial Distributions of Total Gang Crimes}

The first spatial test for our model analyzes how closely agents from every gang engage in crimes throughout the region. Because of boundary and scaling effects, the predicted locations of crimes are slightly skewed, but the pattern of the actual Hollenbeck crimes is qualitatively similar. Using parameters of $\Gamma=0.035, \sigma=2 \sqrt{2}, R^{0}=0.005, \theta=10000, w=0.05, W^{0}=0.000001, C=$ $1, D=0, \delta t=1$, we create a plot (Figure 14) to compare the model to the data. The number of crimes is the same in both subplots.

As Figure 14 shows, our agents appear to fight in similar distances to where the actual Hollenbeck gang members commit crimes. Because modeling total crimes over the 34-month period does not explain activities of individual gangs, we next look at the spatial distribution of crimes for individual gangs.

\subsubsection{Spatial Distributions for Individual Gang Crimes}

Using the agent based model, we generate the locations of individual gang crimes over a period of 1044 days (the same length of time as our actual data). To obtain a better understanding of the data, we compared the distance distributions. Figure 15 displays this spatial analysis for the Opal Street gang. 

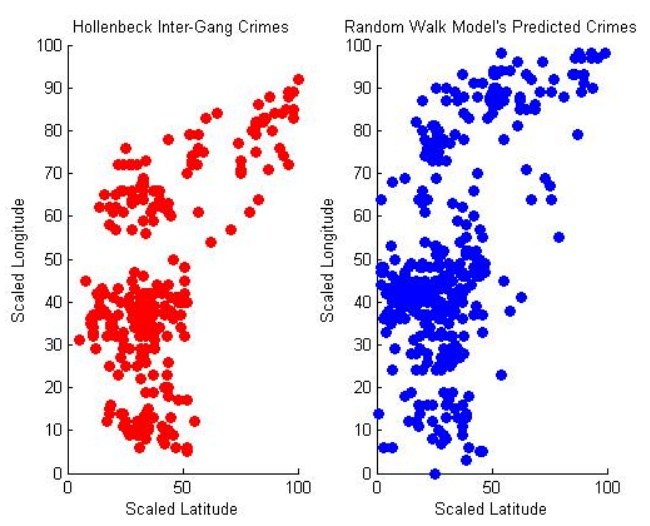

Figure 14: Plot of Hollenbeck total gang crimes (left) from Nov.1999-Sept.2002 versus predicted total gang crimes (right).
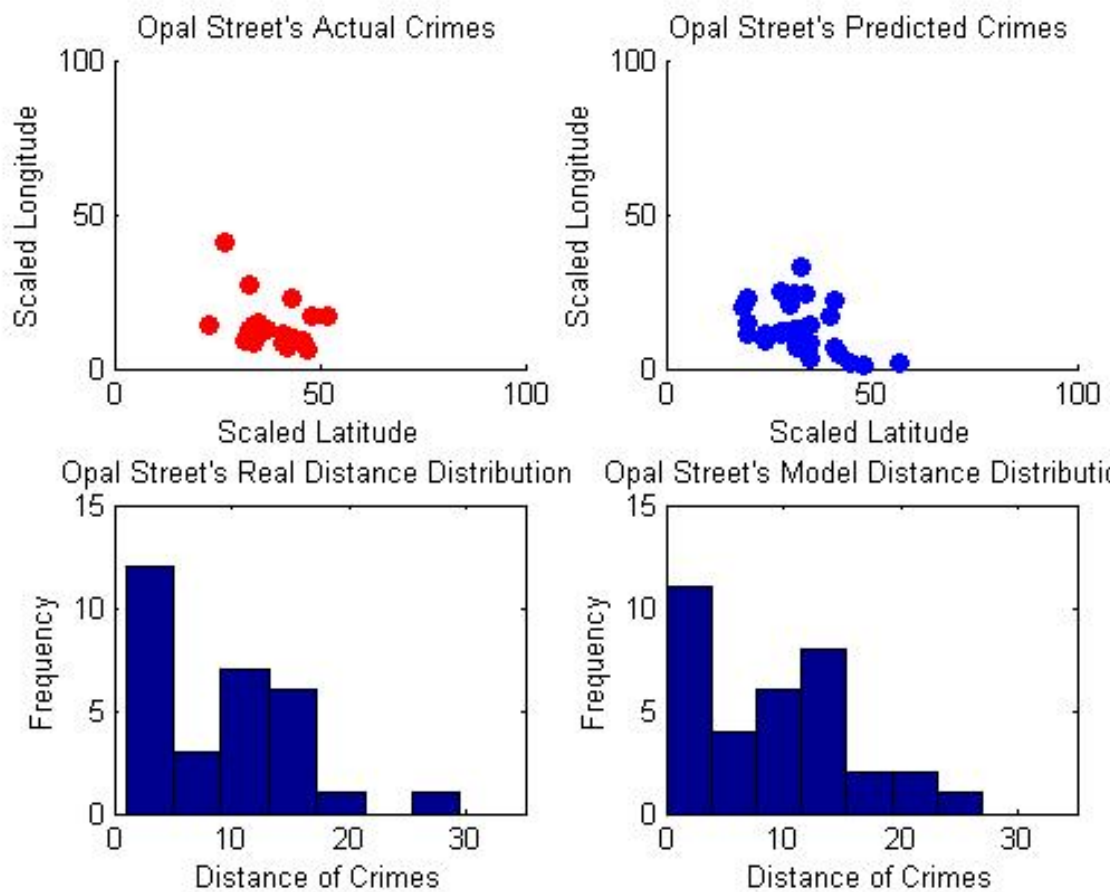

Figure 15: Graph of the Opal Street gang's actual crimes (top left) and our model's predicted attacks for Opal Street(top right). Below are the distance distributions for the plots. 
In Figure 15, the predicted locations of crimes occur in close proximity to where the real Opal Street gang members commit crimes. It follows that the crimes tend to occur at similar distances away from the anchor points; this is evident in the plots of the distance distributions. Next, we analyze how the agent based model predicted the distribution of gang rivalry crimes in space.

\subsubsection{Spatial Distributions for Rivalry Gang Crimes}

The last spatial test using the agent based model analyzes where, in our adjusted Hollenbeck map, an agent commits a crime against a rival gang walker. Figure 16, below, contains plots of the actual 8th Street/VNE rivalry spatial distribution and of the simulated gang rivalry crimes in space.
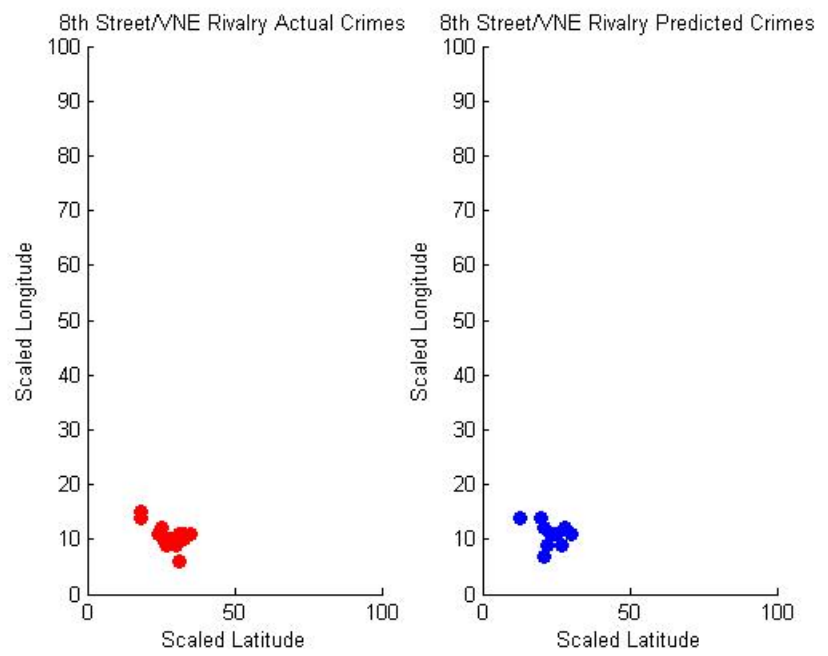

Figure 16: Plot of the 8th Street/VNE rivalry crimes in space (left) against the model's predicted fights between the two gangs (right).

Figure 16 shows that our agent based model accurately predicts where the crimes between the 8th Street and VNE gangs occur.

\subsubsection{Summary}

Spatially, we consider the locations of all the aggregated, individual, and rivalry gang crimes. After noticing the similarities between the actual data and what the agent based model predicted, we can conclude that our simulation fits the data well with respect to crime locations. 


\subsection{Rivalry Analysis}

As gang rivalries are strongly dependent on the distance between gang pairs, a random graph model of gang rivalries must take the distance between nodes into account. One existing model developed for this purpose is Geographic Threshold Graphs $[2,3,4]$ and in this section we compare GTG to graphs generated by the agent based model.

\subsubsection{Adjacency Matrices}

To visualize gang rivalry interactions, we plot the adjacency matrix of the data, a symmetric matrix of 1 s and 0 s. For any entry in the matrix, a 1 corresponds to a connection between the two gangs; the connection is determined by whether or not $n$ crimes occurred. Graphically, this represents a line between the two points. When a threshold $\Omega$ for an adjacency matrix is included, $1 \mathrm{~s}$ are placed in the matrix only when $n \geq \Omega$. Below (Figure 17) is an adjacency matrix that plots the real data.

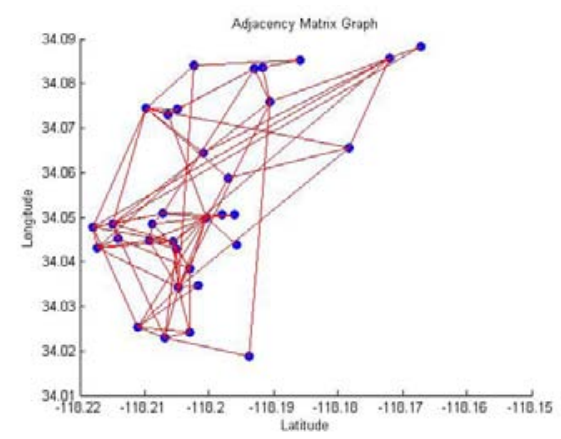

Figure 17: Graph of our Hollenbeck data's adjacency matrix with $\Omega=1$.

Figure 17 displays the adjacency matrix for our data with the nodes in the corresponding relative locations of the anchor points. The axes list the latitude and longitude. We now attempt to simulate these adjacency matrices.

\subsubsection{Agent Based Model}

We create an adjacency matrix from the data produced by the agent based model. Figure 18 displays a simulated adjacency matrix using the same parameters as before. 


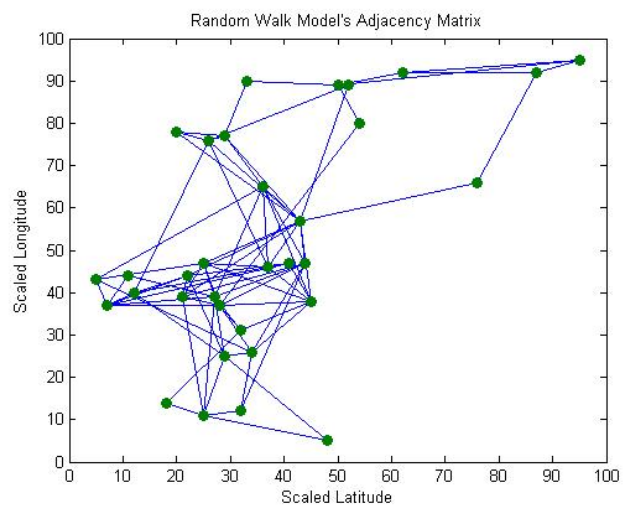

Figure 18: Plot of an agent based model simulated adjacency matrix. This graph has 83 edges, similar to the 82 edges of the actual adjacency matrix.

\subsubsection{Geographical Threshold Graphs}

We can also model gang rivalries by creating an adjacency matrix using Geographical Thresholding. In each simulation, a 1 is placed in an cell of the model's adjacency matrix if the following condition is met:

$$
\frac{g\left(w, w^{\prime}\right)}{r^{\beta}} \geq \theta
$$

where the function $g$ is the interaction strength of the 2 points (anchor points), $w$ and $w^{\prime}$ are the randomly assigned weights of the points, $\mathrm{r}$ is the Euclidean distance between the points, $\beta$ is a parameter of choice (typically $\beta=2$ ) and $\theta$ is the threshold parameter. Found below (Figure 19) is an example of the model alongside an adjacency matrix for the actual data.
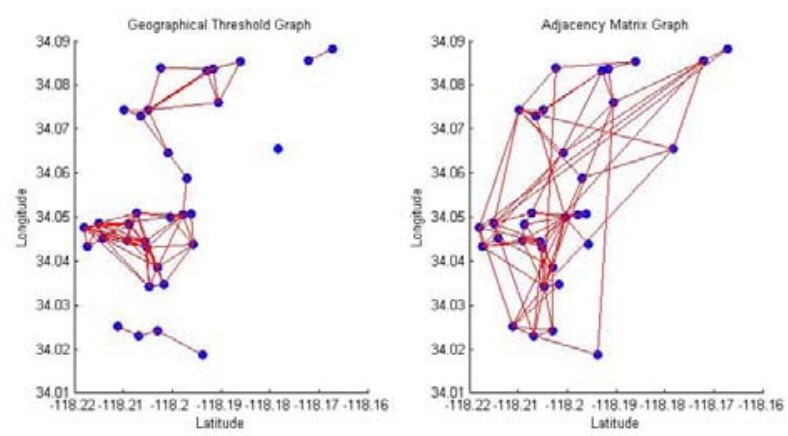

Figure 19: Graph of the 34-month aggregate adjacency matrix $(\Omega=1)$ (right) with the geographical threshold graph (left) 
Figure 19 reveals qualitative differences between the Geographical Threshold Graph and the actual adjacency matrix. Randomly assigning weights is causing the simulation to diverge from the real plot. While the specifics are unclear, the model poorly represents gang rivalries.

\subsubsection{Summary}

The agent based model better models gang rivalry behavior than the geographical threshold graph simulation. Distances between anchor points, previous behavioral patterns, and self-excitation prevent Geographical Thresholding from effectively portraying the Hollenbeck dynamics in our data sample. The agent based model is able to more closely predict the gang interactions by taking into account the behaviors and self-excitation, as well as the distances. Additionally, the agent based model allows gang members to travel further and support more distant rivalries, whereas Geographical Thresholding cannot take into account rivalries over great distances. Thus though distances are important, more properties factor into determining gang rivalries.

\subsection{Statistical Analysis}

\subsubsection{Degree Distributions}

We can test the agent based model by analyzing the actual and predicted degree distributions of the rivalry graphs. The degree corresponds to how many connections one anchor point has with other gangs. Seen below, Figure 20 shows the real plot next to a simulated one.

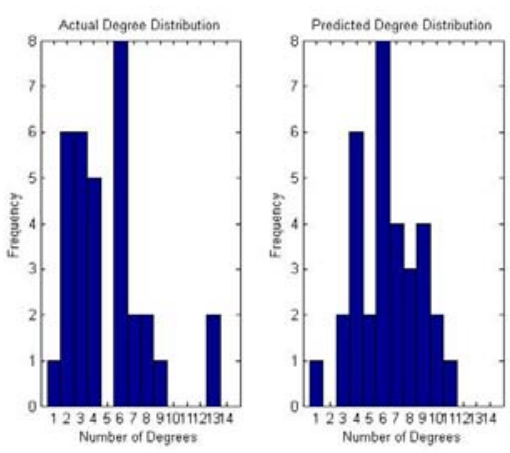

Figure 20: Plot of the 34-month degree distribution (left) next to a generated agent based degree distribution (right)

As seen in Figure 20, the actual data's degree distribution is very irregular, which makes it difficult for any random model to simulate. Despite this, in one specific trial (as seen above), the plot generated by the agent based model reflects the overall shape and most frequently occurring degree. 


\subsubsection{Distance Distributions}

Another way to assess the quality of our model considers the distance distributions. Specifically, we expect our model's agents to commit crimes closer to their anchor point. Figure 21, displaying the distance distributions for the real and the produced data, supports this assumption, despite despite the discrepancies that occur after a distance of 50 scaled units. This similarity further indicates that the agent based model effectively mimics the data.

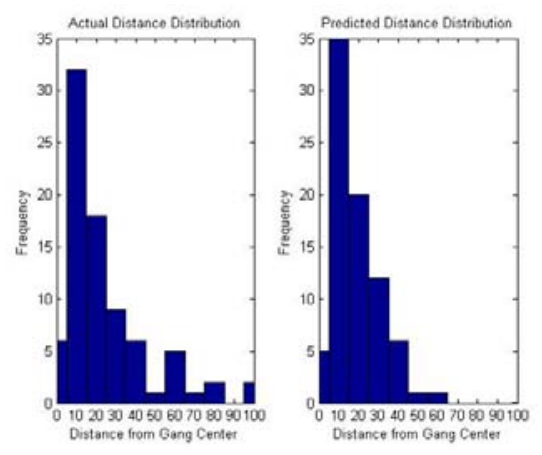

Figure 21: Plot of the actual distance distribution (left) alongside a simulated one (right).

\section{Conclusion}

We are able to confirm that gang-on-gang crimes are better represented by the self-exciting Hawkes process than the memoryless Poisson process. This is done through a histogram analysis of inter-attack times and use of the Akaike Information Criterion. This criterion supports the Hawkes process for six of the nine rivalries with at least ten crimes. The histogram analysis of interattack times agrees with the AIC results. Though not invariably the best fit, the Hawkes process offers an explanation for the clustering of attacks observed in the data.

The agent based model is closely related to the Hawkes process. However, while the Hawkes process simulates rivalry crimes through time, the agent based model mimics both the temporal and spatial distributions of a rivalry's crimes. It may exhibit a wide range of behaviors from completely random motion to motion strongly biased to support rivalries or hotspots. Due to the number of parameters it uses, the agent based model is very flexible and can be given parameters that result in a model that mimics the data well. Because of this flexibility, the agent based model simulates gang rivalries more accurately than Geographical Thresholding.

While the behavior of individual gang members may seem too erratic to predict, the overall network of gangs in Hollenbeck is fairly systematic. Thus, it 
can be well represented by both an agent based model and a self-exciting point process.

In the future it would be beneficial to determine more accurate representations of gang territories and locations for gang anchor points. For example, a more detailed description of gang territories could be obtained by fusing aggregate spatial gang data with existing conceptions of gang geography. Trends in the data indicate that gangs are more likely to be victimized within their territory. This information could be used to improve models and ultimately aid police forces in deterring gang violence.

\section{Acknowledgements}

This work was supported in part by NSF RTG grant DMS-0601395, ARO MURI grant 50363-MA-MUR, NSF grant ACI-0321917, ONR grant N000140810363, NSF grant BCS-0527388, and the Department of Defense. The authors would like to thank Shannon Reid for her role as a mentor and her instruction in ArcGIS, Andrea Bertozzi and George Tita for helpful comments and suggestions, and the LAPD for providing the data used in this study.

\section{References}

[1] Akaike, Hirotugu. A new look at the statistical model identification, IEEE Transactions on Automatic Control 19 (6): 716723 (1974).

[2] Bradonjić, Milan, Aric Hagberg, and Allon G. Percus, The Structure of Geographical Threshold Graphs, Special Issue of Internet Mathematics 4, to appear 2009, available at http://www. ee.ucla.edu/ milan/pubs/BHP_ IM2008.pdf.

[3] Bradonjić, Milan, V. Roychowdhury, and N. Sarshar, General Geographical Threshold Graphs, DIMACS, April 2006, available at http://dimacs. rutgers . edu/Workshops/CombChallenge/slides/bradonjic.pdf.

[4] Bradonjić, Milan, Tobias Müller, and Allon G. Percus, Coloring Geographical Threshold Graphs, SIAM, 2009, available at http://www.siam.org/ proceedings/analco/2009/an109_002_bradonjicm.pdf.

[5] D’Orsogna, M. R., Y. L. Chuang, A. L. Bertozzi, and L. S. Chayes, SelfPropelled Particles with Soft-Core Interactions: Patterms, Stability, and Collapse, Physical Review Letters (2006).

[6] Gang turf in Hollenbeck, Cable News Network LP, LLLP, 2005, available at http://www.cnn.com/CNN/Programs/presents/shows/homicide/ interactive/map/.

[7] Hollenbeck Area Gang Territory Map, Hollenbeck Crime Analysis, 2009. 
[8] Mohler, G.O., M.B. Short, P.J. Brantingham, F.P. Schoenberg, and G.E. Tita, Self-exciting point processes explain spatial-temporal patterns in crime, preprint (2009), available at http://www.math.ucla.edu/ mbshort/papers/crime3.pdf.

[9] Ozaki, T., Maximum Likelihood Estimation of Hawkes' self-exciting point processes, Annals of the Institute of Statistical Mathematics, Volume 31, Number 1, December, 1979.

[10] Short, M.B., M.R. D'Orsogna, P.J. Brantingham, and G.E. Tita, Measuring and modeling repeat and near-repeat burglary effects, accepted in J. Quant. Criminol. (2009), available at http://www.math.ucla.edu/ $\sim$ mbshort/papers/crime2.pdf.

[11] Short, M.B., M.R. D'Orsogna, V.B. Pasour, G.E. Tita, P.J. Brantingham, A.L. Bertozzi, and L.B. Chayes, A statistical model of criminal behavior, Mathematical Models and Methods in Applied Sciences Vol. 18, Suppl. (2008), available at http://www.math.ucla.edu/ mbshort/ papers/crime1.pdf.

[12] Tanner, Herbert G., Ali Jadbabaie and George J. Pappas, Stable Flocking of Mobile Agents, Part I: Fixed Topology, In IEEE Conference on decision and control (2003).

[13] Tita, George Reducing gun violence: results from an intervention in East Los Angeles, RAND Corporation, Santa Monica, CA, 2003.

[14] Tita, George Spatializing Social Networks: Structural Equivalence as a Means to Incorporate Position, Embeddedness, and Place in Social Network Analysis, Annals of the Association of American Geographers.

[15] Vicsek, Tamás, András Czirók, Eshel Ben-Jacob, Inon Cohen, and Ofer Shochet, Novel Type of Phase Transition in a System of Self-Driven Particles, Physical Review Letters Vol. 75 (1995).

[16] Wasserman, Stanley and Katherine Faust, Social Network Analysis: Methods and Applications, Cambridge University Press, New York, NY, 1994. 